\title{
OPEN Neutrophil extracellular traps are induced in a psoriasis model of interleukin-36 receptor antagonist-deficient mice
}

\author{
Soichiro Watanabe ${ }^{1}$, Yohei Iwata ${ }^{1}$, Hidehiko Fukushima ${ }^{1}$, Kenta Saito ${ }^{1}$, Yoshihito Tanaka ${ }^{1}$, \\ Yurie Hasegawa ${ }^{1}$, Masashi Akiyama ${ }^{2}$ \& Kazumitsu Sugiura ${ }^{1 \bowtie}$
}

Loss-of-function mutations in the interleukin (IL)-36 gene IL36RN are associated with psoriasis. The importance of neutrophil extracellular traps (NETs), web-like structures composed of neutrophil DNA, in the pathogenesis of psoriasis has been unclear. Here, we aimed to clarify the role of NET signaling in the deficiency of IL36 receptor antagonist (DITRA). We evaluated the severity of psoriasis-like lesions induced by imiquimod cream treatment in $1 / 36 \mathrm{rn}^{-1-}$ mice. The mRNA levels of psoriasis-related cytokines were measured via real-time reverse transcription polymerase chain reaction, and the effects of $\mathrm{Cl}$-amidine, a peptidyl arginine deiminase 4 (PAD4) inhibitor, on psoriasis-like lesions were evaluated. PAD4 is a histone-modifying enzyme that is involved in NET formation. Psoriasis area and severity index scores, epidermal thickness, and infiltrated neutrophil counts were significantly increased in $/ 136 \mathrm{rn}^{-1-}$ mice; NET formation was confirmed pathologically. Several cytokines and chemokines were upregulated in the skin lesions of $1 / 36 \mathrm{rn}^{-1-}$ mice and $\mathrm{Cl}$-amidine treatment improved these psoriasis-like lesions. These results suggest that NET formation plays an important role in the pathology of psoriasis-like lesions in these mice and might represent a promising therapeutic target for DITRA.

IL36RN encodes interleukin (IL)-36 receptor antagonist (IL-36Ra), an IL-1 cytokine family protein that strictly regulates IL-36 signaling. The IL-36 pathway is induced when IL-36 $\alpha, \beta$, or $\gamma$ binds to its specific receptor, interleukin-1 receptor-related protein 2 (IL-1Rrp2), triggering the recruitment of the co-receptor IL-1 receptor accessory protein (IL-1RacP) and activation of the nuclear factor-kappa B (NF- $\mathrm{BB}$ ), as well as mitogenactivated protein kinase signaling pathways. This enhances the transcription and secretion of pro-inflammatory cytokines $^{1,2}$, which recruit neutrophils, T cells, and dendritic cells (DCs) in the skin. IL-36 stimulates the production of chemotactic agents for activated leukocytes, promoting leukocyte infiltration and acanthosis of the skin ${ }^{3}$. Loss-of-function mutations in IL36RN cause a recessively inherited autoinflammatory keratinization disease known as deficiency of IL-36Ra (DITRA) ${ }^{4-9}$. We previously prepared $I l 36 \mathrm{rn}^{-1-}$ mice and established a DITRA murine model by treating mice with a TLR4 agonist ${ }^{10}$, a severe contact hypersensitivity model by treatment with 1-fluoro-2,4-dinitorobenzene ${ }^{11}$, and a delayed cutaneous wound healing model ${ }^{12}$. It has been known that deficiency of IL36Ra induces severe epidermal proliferation and neutrophil infiltration in imiquimod (IMQ)induced psoriasis-like lesions ${ }^{13,14}$.

The roles of T cells, macrophages, and DCs have been reported in the pathogenesis of psoriasis vulgaris ${ }^{15-19}$. However, the role of neutrophils in this pathology have gradually been clarified ${ }^{20}$ but have not been sufficiently elucidated. Neutrophils play important roles in many diseases, including infectious and neoplastic, autoimmune, and chronic inflammatory disease ${ }^{21-25}$. Upon activation, neutrophils undergo a cell death process known as NETosis, in which nuclear substances are extruded into the extracellular space ${ }^{26,27}$. These structures are named neutrophil extracellular traps (NETs) and are large, web-like structures composed of granule proteins, histones, and decondensed DNA ${ }^{28}$. The existence of NETs has been reported in psoriatic skin, where they might play a role in inducing increased expression of human $\beta$-defensin- $2^{29}$. Therefore, NETs and neutrophils can induce inflammation through various mechanisms, including inflammasome activation ${ }^{30}$, the triggering of Toll-like

${ }^{1}$ Department of Dermatology, Fujita Health University School of Medicine, 1-98 Kutsukake-cho, Toyoake, Aichi 470-1192, Japan. ${ }^{2}$ Department of Dermatology, Nagoya University Graduate School of Medicine, 65 Tsurumai-cho, Showa-ku, Nagoya, Aichi 466-8550, Japan. ${ }^{\bowtie}$ email: ksugiura@fujita-hu.ac.jp 

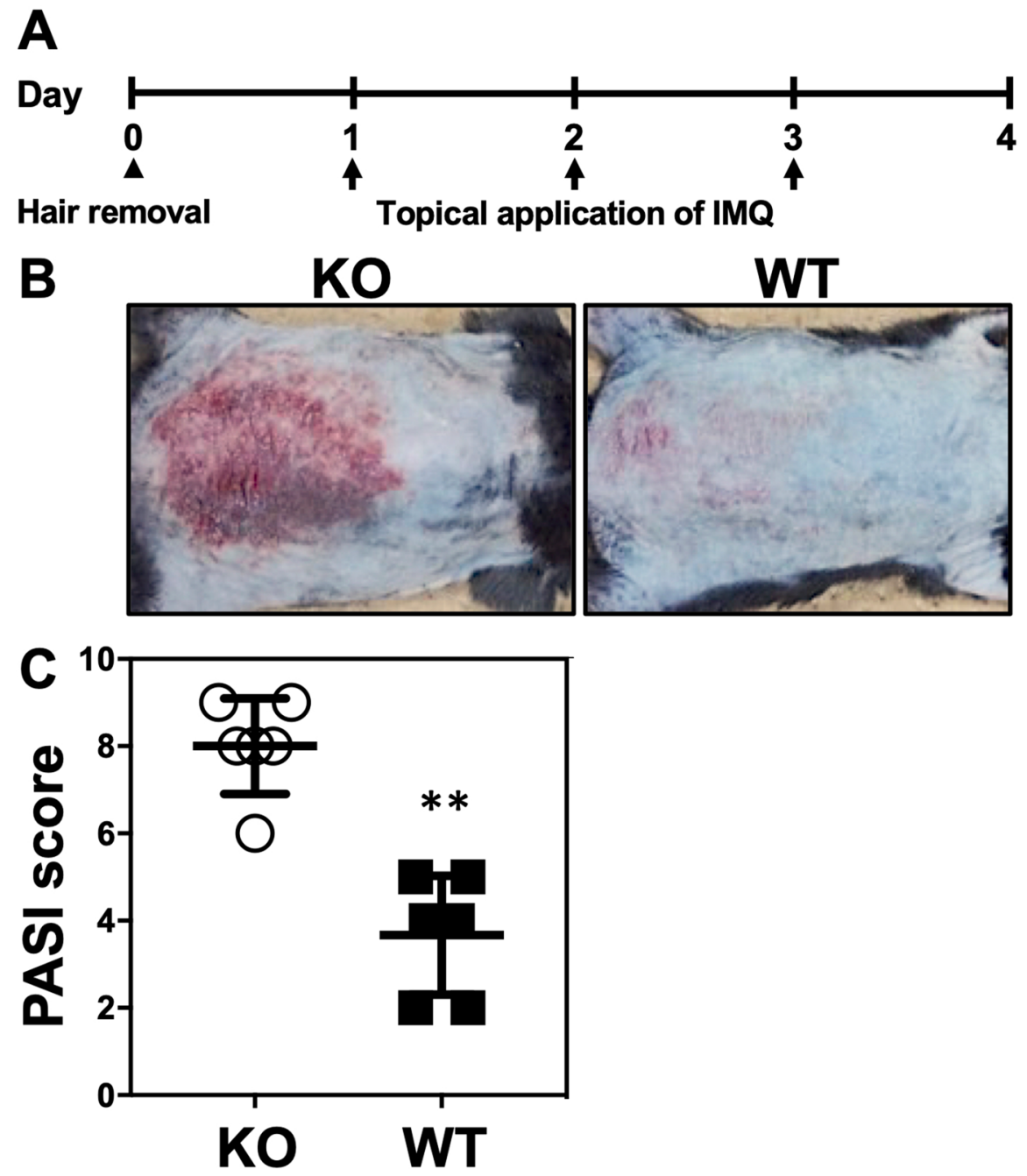

Figure 1. Imiquimod (IMQ) treatment induces psoriasis-like lesions in $\mathrm{Il}_{36 \mathrm{rn}^{-1-}}$ mice. (A) Protocols for the development of IMQ-induced contact psoriasis-like lesions; (B) representative clinical images of back skin (KO: $\mathrm{Il} 36 \mathrm{rn}^{-/-}$mice, WT: wild-type mice). (C) Psoriasis area and severity index (PASI) scores of skin lesions in IMQtreated mice on day $4(\mathrm{n}=6){ }^{* *} p<0.01$.

receptor 7 (TLR7) and TLR9 via self-antigen complexes such as cathelicidin antimicrobial peptide LL37 DNA ${ }^{31}$, macrophage pyroptosis stimulation ${ }^{32}$, and IL-36 cytokine processing and activation ${ }^{33,34}$.

Here, we aimed to clarify the role of NET signaling in DITRA and develop an effective therapy for IMQinduced severe psoriasis in Il36rn ${ }^{-1-}$ mice. As NETs might be involved in DITRA, it could be possible to prevent the development of severe psoriasis-like lesions by prohibiting their formation. Therefore, this study was conducted to determine the immunological pathology associated with severe psoriasis-like lesions in mice with DITRA.

\section{Results}

Estimation of psoriasis area and severity index (PASI) scores and histological characteristics in $/ 136 \mathrm{rn}^{-/-}$mice after consecutive topical application of IMQ cream for 3 days. IMQ treatment for 3 consecutive days induced psoriasis-like lesions in $I l 36 \mathrm{rn}^{-1-}$ mice. For the purpose of assessing the severity of this psoriasis-like lesion, we evaluated the eruption using the PASI score ${ }^{35}$ and compared $\mathrm{Il}_{36 \mathrm{rn}^{-/-}}$and wild-type mice $(\mathrm{n}=6)$. Il36rn $\mathrm{rr}^{-1-}$ mice showed a significant increase in PASI scores (Fig. $\left.1 \mathrm{~A}-\mathrm{C}\right)$. Severe scaling was observed in IMQ-induced $I l 36 \mathrm{rn}^{-1-}$ mice. Next, we pathologically estimated epidermal area, the number of infiltrated neutrophils and NET formation. $\mathrm{Il}_{36 \mathrm{rn}^{-/-}}$mice showed a significant increase in the epidermal area (Fig. 2A,B) compared to that in wild-type mice after IMQ treatment for 3 consecutive days. To evaluate inflammatory cell infiltration in IMQ-induced psoriasis lesions, immunostaining for CD11c, F4/80, CD3, and myeloperoxidase (MPO) was performed. Il36rn ${ }^{-1-}$ mice showed a significant increase in CD11c-, CD3-, and MPO-positive cells compared to those in wild-type mice (Fig. 2C). In addition, to evaluate NET formation in IMQ-induced psoriasis-like lesions, immunofluorescent co-staining for MPO and citrullinated histone H3 was 

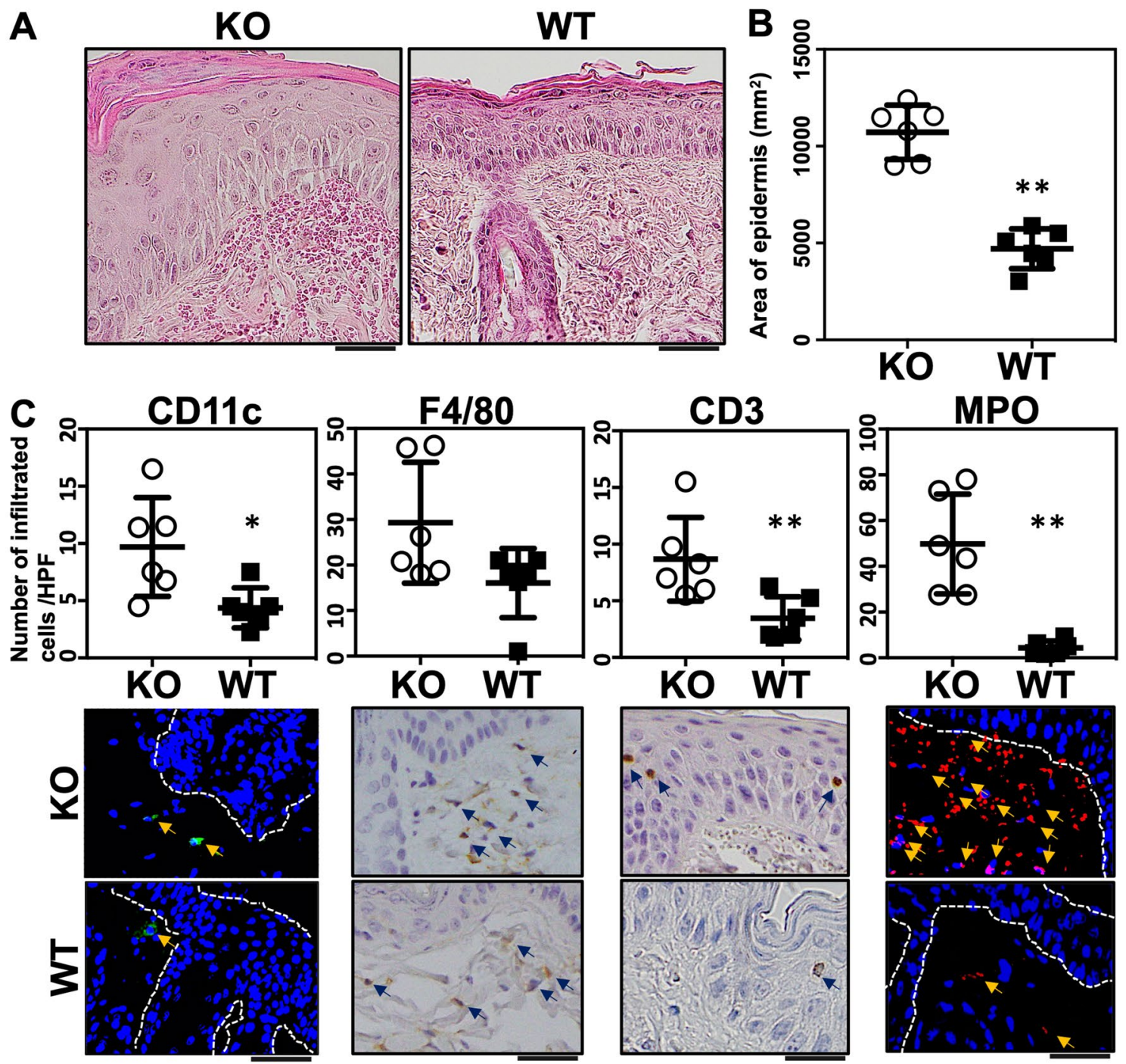

D
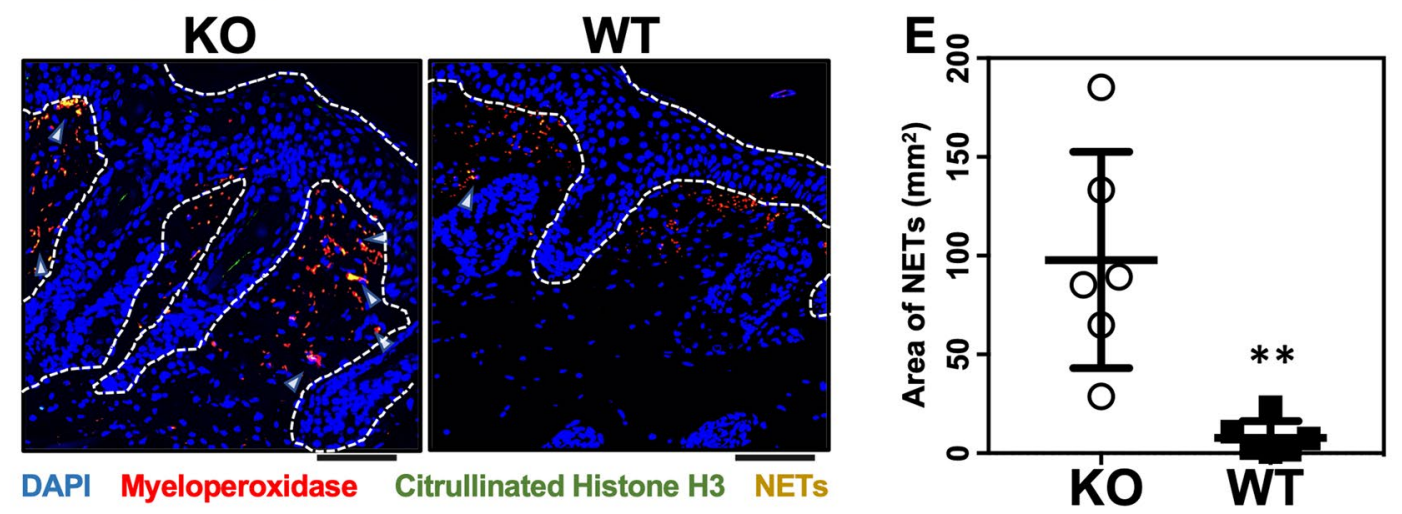

Figure 2. Area of epidermis, numbers of infiltrated neutrophils and neutrophil extracellular trap (NET) formation. (A) Histological images of skin sections from WT and Il36rn ${ }^{-1-}$ mice 3 days after topical imiquimod (IMQ) application. Scale bars, $100 \mu \mathrm{m}$. (B) The epidermal area within a distance of $10 \mathrm{~mm}$ was measured using ImageJ software ( $n=6$ animals/group; ${ }^{* *} p<0.01$ versus WT). (C) Numbers of infiltrated cells/high-power fields in back skin cross-sections from IMQ-treated $\mathrm{Il}_{36 \mathrm{rn}^{-/-}}$and WT mice $(100 \times$ magnification, nine sections per mouse, $\mathrm{n}=6$ animals/group; ${ }^{\star} p<0.05,{ }^{\star *} p<0.01$ versus WT). Representative photos of CD11c, F4/80, CD3, and MPO. Arrow shows positive cells. (D) Enhanced NETosis (arrows) in the dermis of IMQ-treated Il36rn ${ }^{-1-}$ mice. Nuclear staining: 4',6-diamidino-2-phenylindole (blue). Representative immunofluorescence image of NET structures stained with myeloperoxidase (red) and citrullinated histone (green). Scale bar, $50 \mu \mathrm{m}$. (E) Area of NETs/high-power fields in back skin cross-sections from IMQ-treated Il36rn ${ }^{-/}$and WT mice $\left(40 \times\right.$ magnification, nine sections per mouse, Mann-Whitney $U$ test, $n=6$ animals/group; ${ }^{* *} p<0.01$ versus WT). 

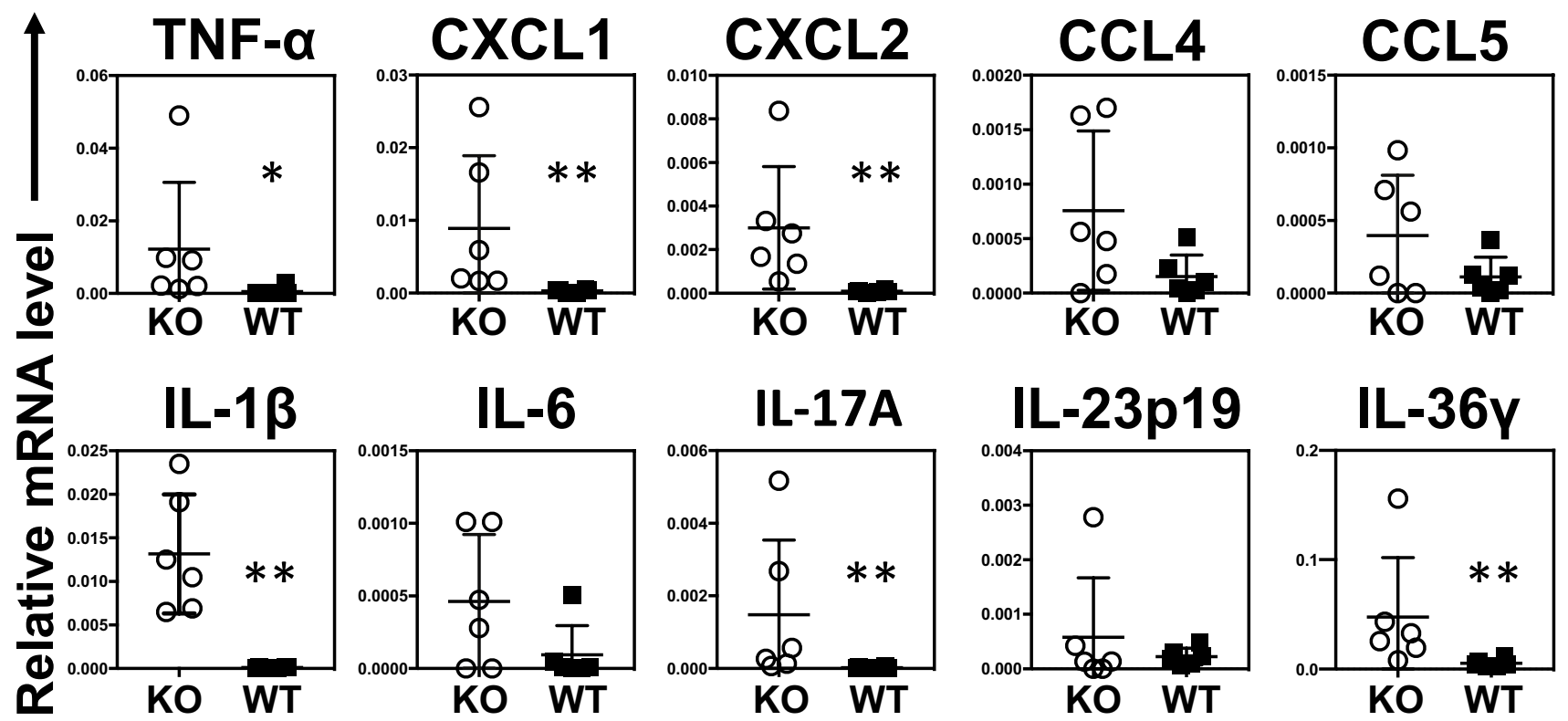

Figure 3. Skin mRNA expression levels. Real-time reverse transcription polymerase chain reaction (RT-PCR) analyses of psoriasis-related cytokines and chemokines from mouse skin samples (Mann-Whitney $U$ test, $n=6$ animals/group ${ }^{\star} p<0.05,{ }^{\star *} p<0.01$ versus WT). Internal control: glyceraldehyde-3-phosphate dehydrogenase $(G A P D H)$ mRNA. KO, IMQ-treated Il36rn ${ }^{-1-}$ mice; WT, IMQ-treated WT mice.

performed. Il36rn ${ }^{-/-}$mice showed a significant increase in the area of NETs compared to that in wild-type mice (Fig. 2D,E). Thus, Il36rn ${ }^{-/-}$mice developed severe IMQ-induced psoriasis-like lesions, increased infiltration of inflammatory cells, and increased NET formation compared to those in wild-type mice.

Chemokine and cytokine expression in psoriasis-like lesions in $/ 136 \mathrm{rn}^{-1-}$ mice. The expression of genes encoding tumor necrosis factor (TNF)- $\alpha, \mathrm{C}-\mathrm{C}$ motif chemokine ligand 4 (CCL4), CCL5, C-X-C motif chemokine ligand 1 (CXCL1), CXCL2, IL-1 $\beta$, IL-6, IL-17A, IL-23p19, and IL-36 $\gamma$ after IMQ treatment for 3 consecutive days was measured via real-time reverse transcription polymerase chain reaction (RT-PCR) in Il36rn ${ }^{-1-}$ and wild-type mice (Fig. 3). Il36rn ${ }^{-1-}$ mice showed significantly increased TNF- $\alpha$, CXCL1, CXCL2, IL-1 $\beta, I L-$ $17 \mathrm{~A}$, and $I L-36 \gamma$ expression levels compared to those in wild-type mice. In contrast, the loss of IL-36Ra did not reach significant difference in a significant difference in CCL4, CCL5, IL-6, or IL-23p19 mRNA expression levels in comparison to those in wild-type mice.

$\mathrm{Cl}$-amidine treatment of IMQ-induced psoriasis in $1 / 36 \mathrm{rn}^{-/-}$mice. NET formation was enhanced in Il36rn ${ }^{-1-}$ mice. Therefore, we hypothesized that NETs would be involved in the pathogenesis of IMQ-induced psoriasis-like lesions. It has been known that $\mathrm{Cl}$-amidine, a pan-peptidyl arginine deaminase (PAD) inhibitor, suppresses NET formation by inhibiting PAD4, an enzyme necessary for NET formation ${ }^{36-39}$. The effects of Clamidine were investigated in IMQ-induced psoriasis-like lesions in $\mathrm{Il}_{36 \mathrm{rn}^{-1-}}$ mice. $\mathrm{Cl}$-amidine $(10 \mathrm{mg} / \mathrm{kg} / \mathrm{day})$ or the same amount of vehicle was subcutaneously administered for 3 days (day 1-3), $4 \mathrm{~h}$ prior to IMQ treatment (Fig. 4A). Interestingly, PASI scores, epidermal area, the number of infiltrated MPO-positive cells, and the area of NETs were significantly reduced by $\mathrm{Cl}$-amidine administration compared with those measured in vehicletreated $I l 36 r^{-1-}$ mice (Fig. 4B,C). In addition, TNF- $\alpha$, CXCL1, IL-1 $\beta, I L-17 A$, and $I L-36 \gamma$ mRNA expression levels in IMQ-induced psoriasis-like lesions were significantly reduced by $\mathrm{Cl}$-amidine administration compared to those in vehicle-treated Il36rn ${ }^{-1-}$ mice (Fig. 4D). CXCL2 $(p=0.0628)$, CCL5 $(p=0.0649)$, and IL-6 $(p=0.0648)$ levels tended to be decreased by $\mathrm{Cl}$-amidine administration compared to those in vehicle-treated $\mathrm{Il} \mathrm{C} 6 \mathrm{rn}^{-1-}$ mice (Fig. 4D). However, there were no significant differences in CCL4 and IL-23p19 expression levels between the $\mathrm{Cl}$-amidine treatment and control groups (Fig. 4D). Thus, blockade of NETosis by $\mathrm{Cl}$-amidine suppressed severe IMQ-induced psoriasis-like lesions in Il36rn ${ }^{-/-}$mice.

In vitro macrophage and NET culture. In our study, $\mathrm{Il}_{36 \mathrm{rn}}{ }^{-1-}$ mice showed significantly increased IL-36 $\gamma$ expression levels compared to those in wild-type mice (Fig. 3). It has been reported that NETs stimulate monocytes and induce secretion of IL-1 $\beta$, IL- $6, \mathrm{IL}-23$, and TNF- $\alpha^{40}$. In addition, the activation of monocytes leads to cell maturation with an increase in Th17 cells, leading to IL-36 $\gamma$ production by keratinocytes through T-cell mediated immune reactions via the IL-17/IL-23/IL-22 $\mathrm{axis}^{40,41}$. Therefore, it is possible that monocytes activated by NETs induce the secretion of IL-36 $\gamma$ through T-cell mediated immune reactions via the IL-17/ IL-23/IL-22 axis. However, it has been reported that macrophages also play an important role in the pathogenesis of psoriasis ${ }^{19}$ and that NETs stimulate IL- $1 \beta$ and IL-18 release by macrophages derived from lupus patients ${ }^{30}$. In addition, it has been reported that NETs promote peritoneal macrophage pyroptosis, a caspase-1-dependent 
A Injection of $\mathrm{Cl}$-amidine $4 \mathrm{~h}$ prior to IMQ treatment
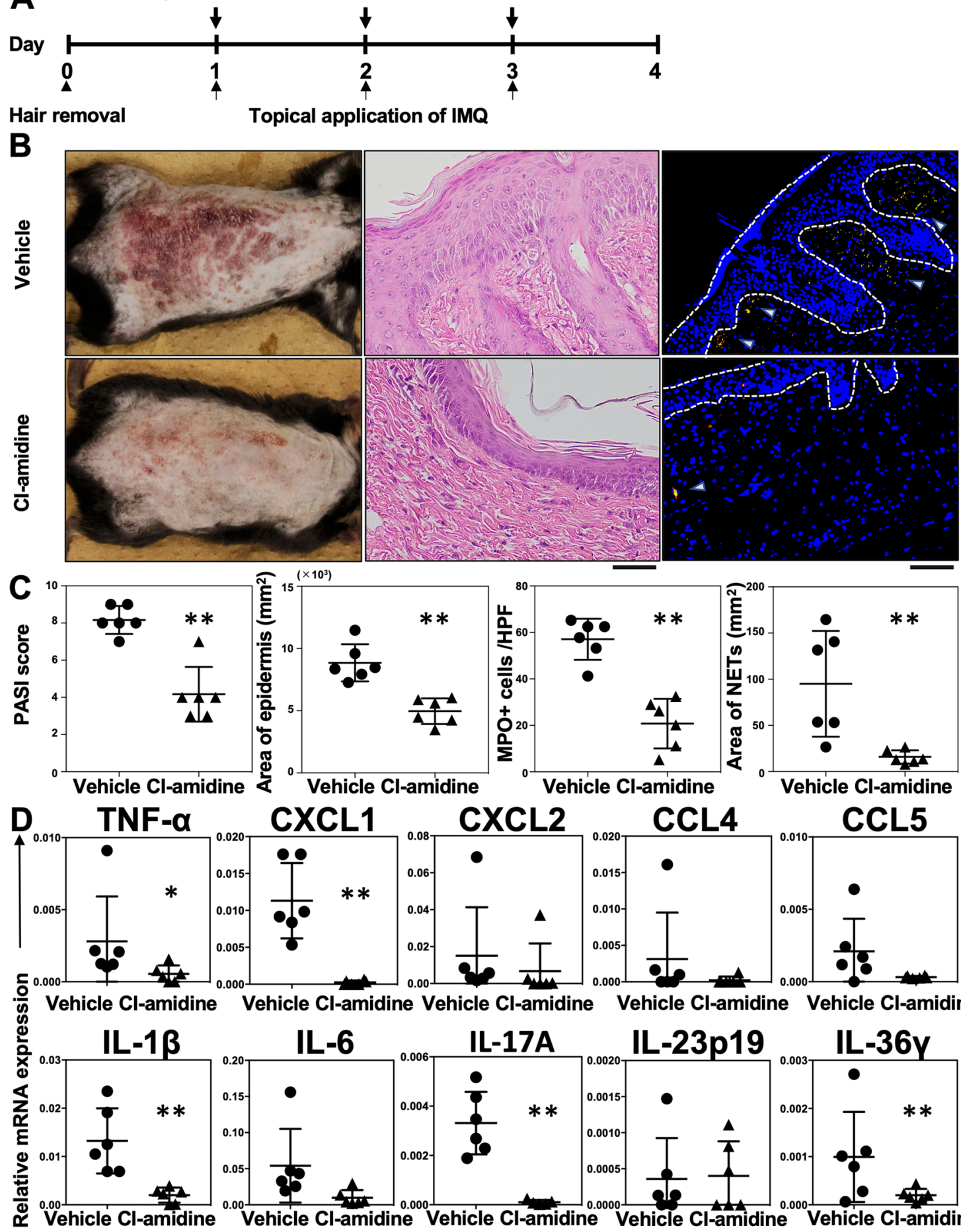

Figure 4. Subcutaneous injection of $\mathrm{Cl}$-amidine decreases psoriasis-like lesions in $\mathrm{Il}_{3} 6 \mathrm{rn}^{-1-}$ mice. (A) Protocols for the development of imiquimod (IMQ)-induced psoriasis-like lesions. For prevention experiments, $\mathrm{Il}_{36 \mathrm{rn}^{-1-}}$ mice were subcutaneously injected with $\mathrm{Cl}$-amidine $(10 \mathrm{mg} / \mathrm{kg}) 4 \mathrm{~h}$ before IMQ treatment (days 1-3). (B) Representative clinical images of back skin and histological images of skin sections from Il36rn ${ }^{-1-}$ mice treated with $\mathrm{Cl}$-amidine or vehicle 3 days after IMQ challenge. Scale bars, $100 \mu \mathrm{m}$. (C) Left: psoriasis area and severity index (PASI) scores of skin lesions in IMQ-challenged mice treated with Cl-amidine or vehicle on day 4 (MannWhitney $U$ test, $\mathrm{n}=6$ animals/group; ${ }^{* *} \mathrm{p}<0.01$ versus vehicle-treated $I l 36 \mathrm{rn}^{-/-}$mice). Second from left: the area of the epidermis within a distance of $10 \mathrm{~mm}$ was measured using ImageJ software. Third from left: number of $\mathrm{MPO}^{+}$cells/high-power field in back skin cross-sections from $\mathrm{Cl}$-amidine- or vehicle-treated $\mathrm{Il}_{36 \mathrm{rn}^{-1-}}$ mice $(100 \times$ magnification, nine sections per mouse, Mann-Whitney $U$ test, $\mathrm{n}=6$ animals/group; ${ }^{* *} p<0.05$ versus vehicle-treated Il36rn ${ }^{-/-}$mice). Right: area of NETs/high-power field in back skin crosssections from $\mathrm{Cl}$-amidine- or vehicle-treated Il36rn ${ }^{-1-}$ mice $(40 \times$ magnification, nine sections per mouse, Mann-Whitney $U$ test, $\mathrm{n}=6$ animals/group; ${ }^{* *} p<0.05$ versus vehicle-treated $\mathrm{Il}_{36 \mathrm{rn}^{-1}}$ mice). (D) Real time RT-PCR analyses of psoriasis-related cytokines and chemokines from mouse skin samples (Mann-Whitney $U$ test, $\mathrm{n}=6$ animals/group ${ }^{*} p<0.05,{ }^{* *} p<0.01$ versus vehicle-treated Il36r ${ }^{-1}$ mice). Data were expressed relative to GAPDH levels. 

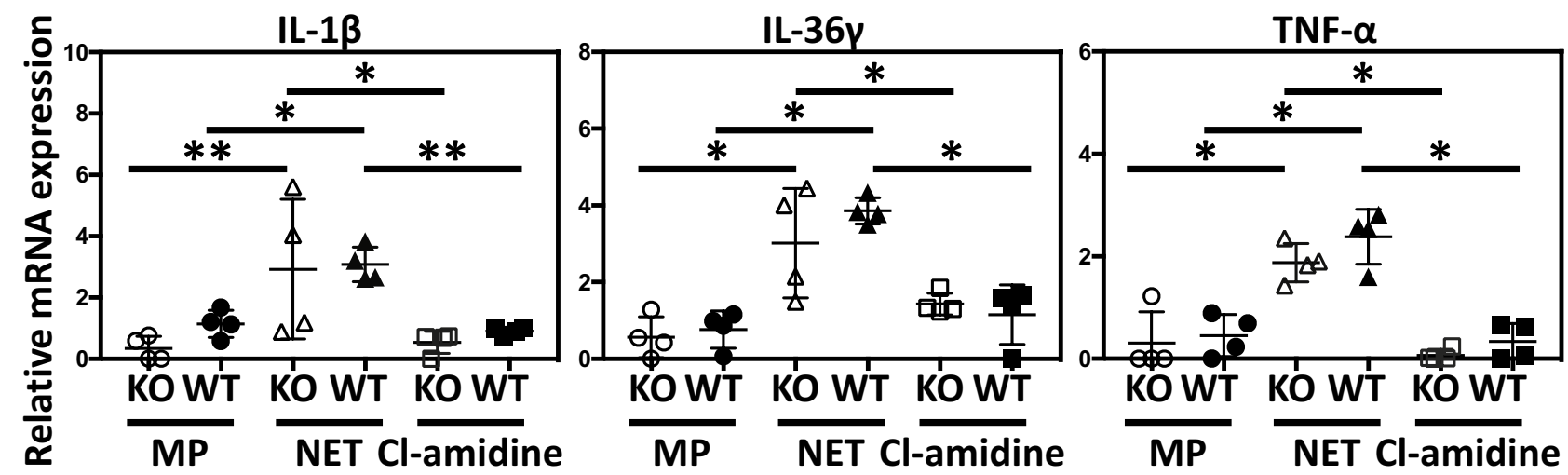

Figure 5. NETs promote the production of psoriasis-related cytokines from macrophages. Real-time RT-PCR analyses of psoriasis-related cytokines from mouse peritoneal macrophages (Mann-Whitney $U$ test, $\mathrm{n}=4$ plates/ group $\left.{ }^{\star} p<0.05,{ }^{* *} p<0.01\right)$. MP: macrophage, NET: macrophage incubated with NETs, Cl-amidine: macrophage incubated with NETs pretreated with Cl-amidine. Data were expressed relative to GAPDH levels.

regulated cell death, and the production of macrophage-derived Il- $1 \beta$ and TNF- $\alpha^{32}$ in sepsis. Furthermore, it is also known that IL-36 produced by macrophages is involved in the pathological condition in psoriasis, rheumatoid arthritis, and Crohn's disease ${ }^{42}$. Therefore, we hypothesized that the increase of IL-36 $\mathrm{\gamma}$ in $\mathrm{Il36 \textrm {rn } ^ { - / - }}$ mice is produced by macrophages activated by NETs. To test this possibility, we performed co-culture of macrophages with NETs, and measured mRNA expression levels of TNF- $\alpha, I L-1 \beta$, and $I L-36 \gamma$, which are involved in the pathological condition of psoriasis.

Peritoneal macrophages of wild-type mice and $I l 36 \mathrm{rn}^{-1-}$ mice were used for in vitro analysis. $I L-1 \beta, I L-36 \gamma$, and TNF- $\alpha$ mRNA expression levels were significantly increased in NET-added macrophages compared to those in macrophages alone, and these mRNA increases were inhibited by pre-treatment of neutrophils with Cl-amidine (Fig. 5). There was no significant difference in mRNA expression levels between macrophages derived from $\mathrm{Il} 36 \mathrm{rn}^{-1-}$ mice and those from wild-type mice. Thus, the production of IL-1 $\beta$, IL-36 $\gamma$, and TNF- $\alpha$ by macrophages was increased by NET stimulation in vitro.

\section{Discussion}

The results of our study demonstrate that IMQ treatment for 3 consecutive days induced psoriasis-like lesions in

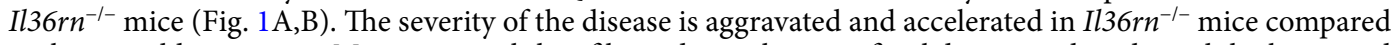
to that in wild-type mice. Many neutrophils infiltrated into the superficial dermis and epidermal thickness and

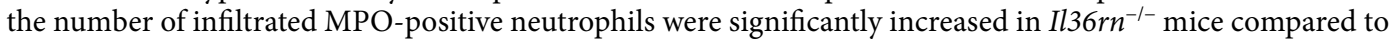
those in wild-type mice (Fig. $2 \mathrm{~A}, \mathrm{~B}, \mathrm{C}$ ), which is consistent with previous studies ${ }^{13}$. In addition, real-time RTPCR analysis showed that $I L-1 \beta, I L-17 A, I L-36 \gamma, C X C L 1$, and CXCL2 expression was increased in IMQ-induced psoriasis_like lesions in Il36rn ${ }^{-1-}$ mice (Fig. 3). As these results were indicative of the global gene expression occurring in the skin, the source of these genetic changes remains unknown. It has been reported that various immune cells and cytokines are involved in the pathogenesis of IMQ-induced psoriasis-like lesions ${ }^{15-20}$. TLR7 stimulation by IMQ causes the production of IL-23 and IL-36 from DCs ${ }^{14}$ and early IL-23 production is caused by IL-36 signaling in keratinocytes ${ }^{43}$. The $\alpha \beta$ T cells or $\gamma \delta$ T cells, which are main producers of IL-17 in IMQinduced psoriasis-like lesions, got activated by IL-23, and secrete IL-17 and IL-22, resulting in the proliferation of keratinocytes ${ }^{14}$. IL-36 derived from DCs stimulates themselves and keratinocytes to further produce IL-36. Additionally, activated keratinocytes produce CXCL1 and CCL20 to facilitate the migration of neutrophils, $\alpha \beta$ $\mathrm{T}$ cells, and $\gamma \delta \mathrm{T}$ cells ${ }^{14}$. Although the presence of $\gamma \delta$ cells was not demonstrated in Il36rn ${ }^{-1-}$ mice, considering previous reports, our results might indicate that stimulation by IL-36 derived from DCs causes the proliferation of keratinocytes and that various chemokines produced by keratinocytes induce the migration of neutrophils, $\alpha \beta$ T cells, and $\gamma \delta$ T cells. However, the difference in IL-23p19 expression in skin lesions did not reach significance in $\mathrm{Il} 36 \mathrm{rn}^{-1-}$ mice compared to that in wild-type mice (Fig. 3). It has been reported that not only IL-23 but also IL-1 $\beta$ induces IL-17 production from $\gamma \delta$ T cells ${ }^{44}$. As $I L-1 \beta$ mRNA levels in skin lesions were significantly elevated in Il36rn $\mathrm{rn}^{-1-}$ mice compared to those in wild-type mice (Fig. 3), the increase in IL-17A in Il36rn ${ }^{-1-}$ mice would be caused mainly by CCL2 0 and IL- $1 \beta$ rather than the IL-23/IL-17/IL-22 axis.

$\mathrm{Il} 36 \mathrm{rn}^{-1-}$ mice showed a significant increase in the number of infiltrated MPO-positive neutrophils (Fig. 2C), which is consistent with the results of previous studies ${ }^{13}$. In addition, the area of NETs was also increased in $I l 36 \mathrm{rn}^{-1-}$ mice compared to that in wild-type mice (Fig. 2E). It has been reported that NETs activate keratinocytes and promote the production of LCN2, IL-36 $\gamma$, CXCL8, and CXCL1 ${ }^{20}$. Endogenous neutrophil-derived TLR4 ligands synergize with IL-36, signaling through MyD88 and NF- $\kappa B$ activation, to induce LCN2 and IL-36 $\gamma$ production. In turn, the upregulated LCN2 modulates NET formation and neutrophil migration, enhancing and sustaining the inflammatory response ${ }^{20}$. In accordance with a previous report, IL-36 $\gamma$ and CXCL1 were increased in IMQ-induced psoriasis-like lesions in Il36rn ${ }^{-1-}$ mice, abundant with NETs (Fig. 3). Next, we examined the effect of Cl-amidine, which is a pan-PAD inhibitor, in IMQ-induced psoriasis-like lesions of $\mathrm{Il36rn}^{-/-}$mice. The administration of $\mathrm{Cl}$-amidine significantly inhibited NET formation in $\mathrm{Il}_{36 \mathrm{rn}^{-1-}}$ mice. In addition, PASI scores, the epidermal area, and the number of infiltrated MPO-positive cells were significantly reduced by Cl-amidine 


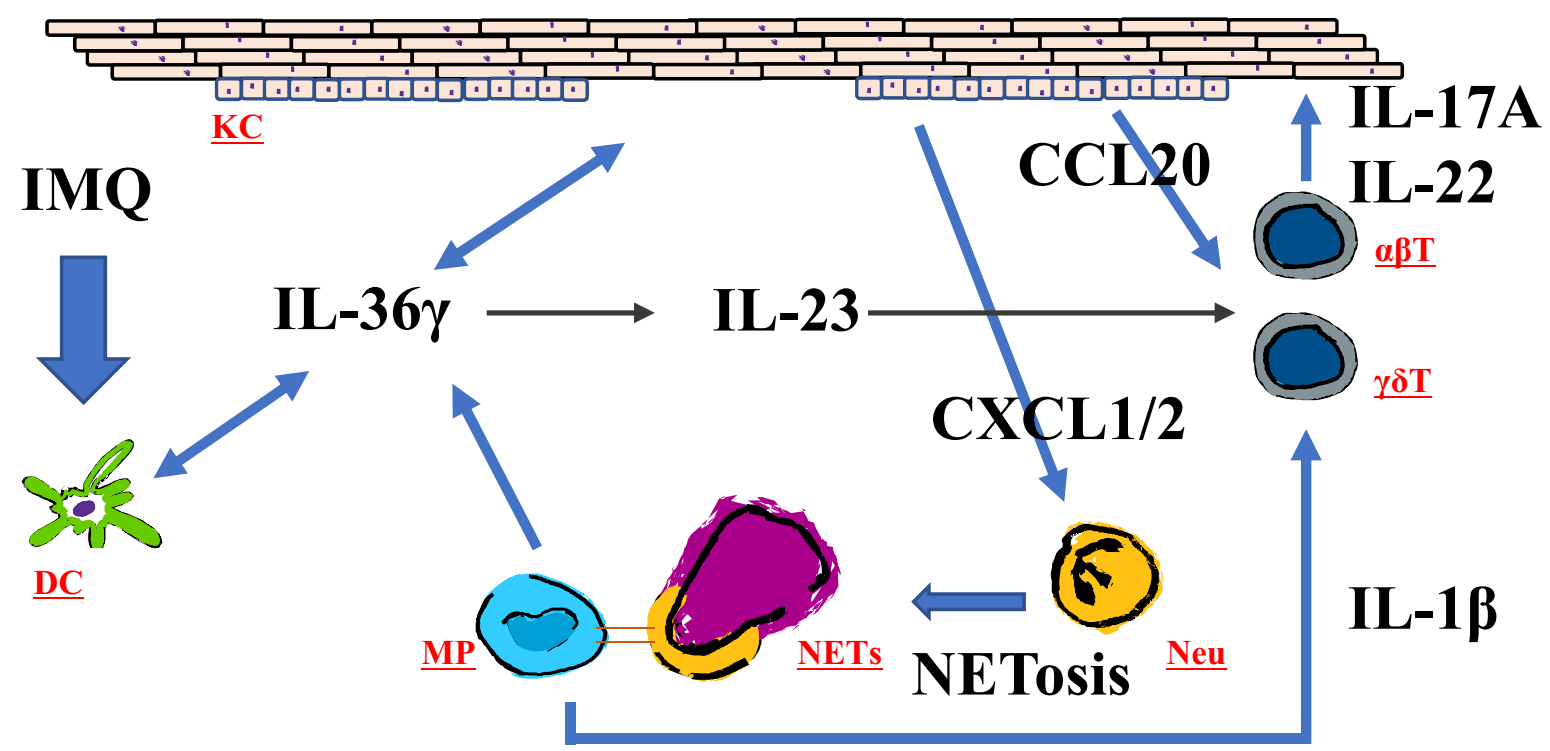

Figure 6. Scheme illustrating pathology of psoriasis-like lesions. Neutrophils cause NETosis, and cytokines, including IL-1 $\beta$ and IL-36 $\gamma$ are secreted by macrophages activated by NETs, and the production of IL-17A from $\gamma \delta$ T cells is promoted by IL-1 $\beta$ or CCL20 from imiquimod (IMQ)-induced psoriasis-like lesions. KC: keratinocytes DC: dendritic cells MP: macrophages NETs: neutrophil extracelluar traps Neu: neutrophils. $\alpha \beta T$ : $\alpha \beta \mathrm{T}$ cells $\gamma \delta \mathrm{T}: \gamma \delta \mathrm{T}$ cells.

administration compared with those measured in vehicle-treated $I l 36 r n^{-1-}$ mice (Fig. $4 \mathrm{~B}$ and C). Furthermore, $\mathrm{Cl}$-amidine-treated Il36rn $\mathrm{rm}^{-1-}$ mice with psoriasis-like lesions showed decreased TNF- $\alpha$, CXCL1, IL-1 $\beta$, IL-36 $\gamma$, and IL-17A expression compared to those in untreated, IMQ-induced Il36rn ${ }^{-1-}$ mice. Thus, the inhibition of NET formation improved IMQ-induced psoriasis-like lesions in $I l 36 \mathrm{rn}^{-1-}$ mice. Since Cl-amidine is a pan-PAD inhibitor, it is therefore possible that the effect of Cl-amidine on $\mathrm{Il}_{36 \mathrm{rn}^{-1-}}$ mice is due to the inhibition of PADs other than PAD4. PAD1 mutations have been associated with the severity of psoriasis in patients ${ }^{45}$, and PAD1 is importantly expressed in skin keratinocytes and hair follicles ${ }^{46}$. Although the effect of Cl-amidine could affect PAD1 in skin keratinocytes and hair follicles, Shao et al. also reports that the administration of Cl-amidine to IMQ-induced psoriasis-like lesions is as effective as DNase I, which breaks down NETs, in scaling, acanthosis, and inflammatory infiltration ${ }^{20}$. As this information is merely comparison of the effect between Cl-amidine and DNase I, it strongly suggests that the inhibition of PAD4 by Cl-amidine suppressed NET formation and improved IMQ-induced psoriasis-like lesions in Il36 $\mathrm{rn}^{-1-}$ mice. Collectively, increased MPO-positive neutrophil infiltration and NET formation in $I l 36 \mathrm{rn}^{-/-}$mice would play a central role in the pathogenesis of IMQ-induced psoriasis-like lesions.

NETs promote macrophage pyroptosis and the production of macrophage-derived Il- $1 \beta$ and TNF- $\alpha^{32}$. In addition, it has been reported that macrophages and monocytes secrete various cytokines such as IL-36 ${ }^{42}$. Therefore, we investigated the changes in IL-1 $\beta$, IL-36 $\gamma$, and TNF- $\alpha$ production by macrophages upon NET stimulation. $I L-1 \beta, I L-36 \gamma$, and TNF- $\alpha$ mRNA expression levels were significantly increased in NET-added macrophages compared to those in macrophages alone (Fig. 5). These mRNA increases were inhibited by pre-treatment of $\mathrm{Cl}$-amidine (Fig. 5). These results suggest that various cytokines involved in the pathogenesis of IMQ-induced psoriasis-like lesions would be produced not only by keratinocytes but also by macrophages and that NETs could promote the production of these cytokines.

Based on our results and previous reports, the mechanisms of IMQ-induced psoriasis-like lesions in $\mathrm{Il} 36 \mathrm{rn}^{-1-}$ mice are illustrated in Fig. 6. DCs activated by IMQ secrete IL-36 $\gamma$, causing the proliferation of keratinocytes. CCL20 generated from the keratinocytes mainly enhances the migration of $\gamma \delta \mathrm{T}$ cells and the secretion of a large amount of IL-17A. Neutrophils can cause NETosis, and cytokines, including IL-1 $\beta$, IL-36 $\gamma$, and TNF- $\alpha$, are secreted by macrophages activated by NETs; moreover, MyD88/NF-kB activation by IL-36 $\gamma$ and the production of IL-17A from $\gamma \delta$ T cells promoted by IL- $1 \beta$ form a pathological condition of IMQ-induced psoriasis-like lesions.

In summary, our results suggest that IL-36Ra loss causes the development of severe psoriasis-like lesions after IMQ treatment for 3 consecutive days by increasing the infiltration of neutrophils into the skin, which is associated with the activation of IL-36R-mediated sustained inflammatory signaling. NET formation also contributes to the development of severe psoriasis-like lesions in $\mathrm{Il}_{36 \mathrm{rn}^{-/-}}$mice. Therefore, NETs may represent promising therapeutic targets for DITRA.

\section{Methods}

Mice. Il36rn ${ }^{-1-}$ mice were generated as previously reported ${ }^{11}$. All experiments were repeated twice using fertile and healthy mice that displayed no evidence of disease or infection. All mice were housed in a pathogen-free barrier establishment and were screened regularly for pathogens. Male C57BL/6 N (Charles River Laboratories, Inc., Wilmington, MA, USA) mice aged 8-12 weeks were used in this study. All studies and procedures were 
approved by the Animal Care and Use Committee of Fujita Health University (AP16113) and performed in accordance with the National Institutes of Health guide for the care and use of laboratory animals.

Psoriasis model in mice. The back skin of male wild-type or $136 \mathrm{rn}^{-1-}$ mice was shaved using an electric clipper and depilatory cream before treatment. To establish a mouse model of psoriasis, wild-type and $I L 36 \mathrm{rn}^{-/-}$ mice were topically administered IMQ cream (62.5 mg Beselna Cream, containing 5\% IMQ; kindly gifted by MOCHIDA PHARMACEUTICAL CO., LTD., Tokyo, Japan) on the back skin for 3 consecutive days over an area of $3 \times 2 \mathrm{~cm}$ (Fig. 1A). In some experiments, Cl-amidine $(10 \mathrm{mg} / \mathrm{kg} / \mathrm{day}$, Cayman Chemical Company, Ann Arbor, MI, USA), a PAD4 inhibitor, was subcutaneously injected.

PASI assessment. The severity of skin lesions was graded according to PASI, which assesses skin erythema, scaling, and thickness. PASI scores were $0-4$, as follows: 0 , none; 1 , slight; 2 , moderate; 3 , marked; 4 , severe ${ }^{35}$. The mice in each group were assessed on day 4.

Histological assessment. Mice were sacrificed via cervical dislocation, and the fresh back skin of each mouse was harvested. Fresh mouse skin samples were fixed in $4 \%$ paraformaldehyde solution for $24 \mathrm{~h}$, dehydrated, and embedded in paraffin. Paraffin sections were stained with hematoxylin and eosin. Epidermal thickness was evaluated by measuring the area of the epidermis. The area of the epidermis within a distance of $10 \mathrm{~mm}$ was measured using ImageJ software (version 1.53; NIH, Bethesda, MD, USA, https://imagej.nih.gov/ij/) ${ }^{47}$. Neutrophil infiltration was assessed by counting the number of neutrophils in nine high-power fields per section. Each section was examined independently by two investigators in a blinded manner, and the means of these measurements were used for analysis.

Tissue immunofluorescence and immunohistochemical staining. For immunofluorescence staining, the skin sections were fixed, stained, and imaged via confocal microscopy. Embedded tissues were cut into 5 - $\mu \mathrm{m}$ sections and washed using phosphate-buffered saline (PBS). These sections were treated with blocking solution (normal donkey serum diluted 1:19 in buffer: $\mathrm{PBS}+1 \%$ bovine serum albumin) for $30 \mathrm{~min}$ at $27^{\circ} \mathrm{C}$. To evaluate the location of NETs, mouse skin tissues were incubated with specific primary antibodies for MPO (1:100, Research and Diagnostic Systems, Inc., Minneapolis, MN, USA) and citrullinated-histone H3 (1:250, Abcam, Cambridge, UK) for $2 \mathrm{~h}$ at $27^{\circ} \mathrm{C}$. The sections were then washed with PBS and incubated with secondary antibodies (donkey anti-goat immunoglobulin G (IgG), Novus Biologicals, Littleton, CO, USA; Alexa Fluor 488, Thermo Fisher SCIENTIFIC, Waltham, MA, USA; donkey anti-rabbit IgG, Novus Biologicals; and Alexa Fluor 647, Thermo Fisher SCIENTIFIC) for $30 \mathrm{~min}$ at $27^{\circ} \mathrm{C}$. The sections were then enclosed in Fluoroshield mounting medium with 4',6-diamidino-2-phenylindole (Abcam). Confocal images were acquired using an Olympus Fluoview 1000 microscope (Olympus Life Science, Tokyo, Japan) with a PlanApo N ( $\times 40$ with and without 2.5 digital zoom). Immunofluorescence of CD11c (1:200, Abcam) was also performed and Alexa Fluor 647 was used as a secondary antibody.

For immunohistochemistry of mouse samples, 6 - $\mu$ m-thick sections of paraffin-embedded tissues were cut, deparaffinized in xylene, and rehydrated in phosphate buffered saline (PBS). Deparaffinized sections were treated with endogenous peroxidase blocking solution (horse serum diluted 1:1 in buffer: PBS + bovine serum albumin $1 \%$ ) for $15 \mathrm{~min}$ at room temperature. Sections were then incubated overnight at $4{ }^{\circ} \mathrm{C}$ with rabbit monoclonal antibodies (mAb) specific for CD3 (1:500, Cell Signaling Technology, Inc., Tokyo, Japan), and F4/80 (1:800, Cell Signaling Technology, Inc.). Sections were then washed in PBS buffer and biotin-conjugated secondary antibodies were applied, followed by incubation with VECTASTAIN Elite ABC Kit (Vector Laboratories, Inc., Burlingame, CA, USA) for $30 \mathrm{~min}$ at room temperature and three washes with PBS for $15 \mathrm{~min}$ each. Peroxidase activity was observed using an ImmPACT DAB Substrate Kit (Vector Laboratories, Inc.) and samples were counterstained with hematoxylin. For a negative control, primary antibody was not added to the sections.

RNA extraction and real-time RT-PCR. Qiagen RNeasy spin columns (QIAGEN, Valencia, CA, USA) was used to extract total RNA from the mouse back skin tissue samples ${ }^{11}$. The total RNA was reverse-transcribed into cDNA using a Prime Script RT Reagent Kit (Takara Bio Inc., Otsu, Japan). Expression levels of genes encoding IL-1 $\beta$, IL-6, IL-17A, IL-36 $\gamma$, CCL4, CCL5, CXCL1, CXCL2, TNF- $\alpha$, and IL-23p19 were measured via realtime RT-PCR using a Light Cycler System (F. Hoffman-La Roche, Ltd, Basel, Switzerland) ${ }^{11}$. PCR samples were prepared in microcapillary tubes as $20-\mu \mathrm{L}$ reactions containing diluted cDNA solution $(2.0 \mu \mathrm{L})$, and the PCR program was performed according to the manufacturer's instructions. Glyceraldehyde-3-phosphate dehydrogenase $(G A P D H)$ expression was used as a standard against which the relative mRNA expression levels of different target genes were calculated using the $2^{-\Delta \Delta \mathrm{Ct}}$ method. Primer sequences used for each gene were provided with the pre-verified Primetime qPCR Assay (Integrated DNA Technologies, Inc., Coralville, IA, USA) ${ }^{12}$.

In vitro experiments. Collection of peritoneal cells from the peritoneal cavities of mice. Mice were intraperitoneally injected with cold PBS $(10 \mathrm{~mL})$. The abdomen was massaged to suspend the cells, and $8 \mathrm{~mL}$ PBS was collected. The cells obtained were centrifuged at $500 \times g$ at $4{ }^{\circ} \mathrm{C}$ for $10 \mathrm{~min}$. Red blood cell lysis buffer was added, and the cells were again centrifuged at $500 \times g$ at $4{ }^{\circ} \mathrm{C}$ after incubation at $4{ }^{\circ} \mathrm{C}$ for 10 min to obtain the peritoneal cells ${ }^{48}$.

Collection of neutrophils from the peritoneal cavities of mice. Neutrophils were collected from the peritoneal cavities of mice using a Neutrophil Isolation Kit (Cayman Chemical Company) according to the manufacturer's 
protocol. Casein $(7.5 \%, 1 \mathrm{~mL})$ was injected into the peritoneal cavity of mice followed by neutrophil isolation medium ( $5 \mathrm{~mL}, \mathrm{PBS}+1 \% \mathrm{BSA}$ ) $24 \mathrm{~h}$ later. The abdomen was massaged to suspend the cells, and the medium $(4 \mathrm{~mL})$ was collected. The fluid from the peritoneal cavity was slowly layered onto $63 \%$ Percoll solution, which was then centrifuged at $1,000 \times g$ at $27^{\circ} \mathrm{C}$ for $20 \mathrm{~min}$. The Percoll solution was aspirated, and PBS with BSA $(9 \mathrm{~mL})$ was added. The samples were centrifuged at $500 \times g$ at $27^{\circ} \mathrm{C}$ for $5 \mathrm{~min}$. Red blood lysis buffer $(5 \mathrm{~mL})$ was added, and the samples were centrifuged at $500 \times \mathrm{g}$ at $27^{\circ} \mathrm{C}$ for $10 \mathrm{~min}$ after incubation at $27^{\circ} \mathrm{C}$ for $10 \mathrm{~min}$ to obtain the mouse neutrophils.

NET generation. For NET generation, $1 \times 10^{6}$ neutrophils were seeded into 24 -well plates in serum-free Dulbecco's modified Eagle's medium (DMEM) and then stimulated to release NETs by adding phorbol 12-myristate 13-acetate (50 nM; Sigma-Aldrich Co. LLC., St Louis, MO, USA) for $4 \mathrm{~h}$ at $37^{\circ} \mathrm{C}$. The medium was removed, and the cell layer was carefully washed with PBS $(2 \mathrm{~mL})$. The PBS was collected after vigorous agitation and centrifuged at $500 \times g$ at $4{ }^{\circ} \mathrm{C}$ for $10 \mathrm{~min}$. Cell-free NET structures were then collected in the supernatant phases ${ }^{20}$. Some neutrophils were pretreated with $\mathrm{Cl}$-amidine $(10 \mu \mathrm{M}$; Cayman Chemical Company) before NET generation for $2 \mathrm{~h}$ at $37^{\circ} \mathrm{C}^{49}$.

Macrophage culture. Peritoneal cells (mixture of macrophages, B cells, and T cells, $0.5 \times 10^{6}$ ) were cultured in 24-well plates in serum-free DMEM. After $24 \mathrm{~h}$, non-adherent cells (B cells and T cells) were removed by gently washing three times with warm $\mathrm{PBS}^{48}$. In some experiments, isolated macrophages were co-cultured with NETs originated from $0.5 \times 10^{6}$ neutrophils. After $12 \mathrm{~h}$ of culture, total RNA was extracted and subsequently reversetranscribed.

Statistical analysis. Data were analyzed using a GRAPHPAD PRISM software (version 7, Graph Pad Software, La Jolla, CA, USA, https://www.graphpad.com/scientific-software/prism/) and presented as means \pm standard deviations. For comparisons between groups, Mann-Whitney $U$ tests and one-way analysis of variance were used. Values of $p<0.05$ were considered to indicate statistical significance.

\section{Data availability}

The datasets generated and/or analyzed during the current study are available from the corresponding author upon reasonable request.

Received: 7 May 2020; Accepted: 3 November 2020

Published online: 19 November 2020

\section{References}

1. Towne, J. E., Garka, K. E., Renshaw, B. R., Virca, G. D. \& Sims, J. E. Interleukin (IL)-1F6, IL-1F8, and IL-1F9 signal through IL1Rrp2 and IL-1RAcP to activate the pathway leading to NF-kappaB and MAPKs. J. Biol. Chem. 279, 13677-13688 (2004).

2. Dinarello, C. et al. IL-1 family nomenclature. Nat. Immunol. 11, 1-3 (2010).

3. Foster, A. M. et al. IL-36 promotes myeloid cell infiltration, activation and inflammatory activity in skin. J. Immunol. 192, 60536061 (2014).

4. Marrakchi, S. et al. Interleukin-36-receptor antagonist deficiency and generalized pustular psoriasis. N. Engl. J. Med. 365, 620-628 (2011).

5. Onoufriadis, A. et al. Mutations in IL36RN/IL1F5 are associated with the severe episodic inflammatory skin disease known as generalized pustular psoriasis. Am. J. Hum. Genet. 89, 432-437 (2011).

6. Sugiura, K. et al. The majority of generalized pustular psoriasis without psoriasis vulgaris is caused by deficiency of interleukin-36 receptor antagonist. J. Invest. Dermatol. 133, 2514-2521 (2013).

7. Sugiura, K. The genetic background of generalized pustular psoriasis: IL36RN mutations and CARD14 gain-of-function variants. J. Dermatol. Sci. 74, 187-192 (2014).

8. Akiyama, M., Takeichi, T., McGrath, J. A. \& Sugiura, K. Autoinflammatory keratinization diseases: An emerging concept encompassing various inflammatory keratinization disorders of the skin. J. Dermatol. Sci. 90, 105-111 (2018).

9. Akiyama, M., Takeichi, T., McGrath, J. A. \& Sugiura, K. Autoinflammatory keratinization diseases. J. Allergy Clin. Immunol. 140, 1545-1547 (2017).

10. Shibata, A. et al. Toll-like receptor 4 antagonist TAK-242 inhibits autoinflammatory symptoms in DITRA. J. Autoimmun. 80, 28-38 (2017).

11. Fukushima, H. et al. TAK-242 ameliorates contact dermatitis exacerbate by IL-36 receptor antagonist deficiency. Sci. Rep. 10, 1-10 (2020).

12. Saito, K. et al. IL-36 receptor antagonist deficiency resulted in delayed wound healing due to excessive recruitment of immune cells. Sci. Rep. 10, 1-12 (2020).

13. Palomo, J., Troccaz, S., Talabot-Aye, D., Rodriguez, E. \& Palmer, G. The severity of imiquimod-induced mouse skin inflammation is independent of endogenous IL-38 expression. PLoS ONE 13, 1-15 (2018).

14. Tortola, L. et al. Psoriasiform dermatitis is driven by IL-36-mediated DC-keratinocyte crosstalk. J. Clin. Invest. 122, 3965-3976 (2012).

15. Bagchi, S. et al. CD1b-autoreactive T cells contribute to hyperlipidemia-induced skin inflammation in mice. J. Clin. Invest. 127, 2339-2352 (2017).

16. Di Meglio, P. et al. Targeting CD8(+) T cells prevents psoriasis development. J. Allergy Clin. Immunol. 13, 274-276 (2016).

17. Kirby, B. Langerhans cells in psoriasis: Getting to the core of the disease. Br. J. Dermatol. 178, 1240 (2018).

18. Wang, H. et al. Activated macrophages are essential in a murine model for T cell-mediated chronic psoriasiform skin inflammation. J. Clin. Invest. 116, 2105-2114 (2006).

19. Boehncke, W. H. \& Schön, M. P. Psoriasis. Lancet 386, 983-994 (2015).

20. Shao, S. et al. Neutrophil extracellular traps promote inflammatory responses in psoriasis via activating epidermal TLR4/IL-36R crosstalk. Front.. Immunol. 10, 1-14 (2019).

21. Park, J. et al. Cancer cells induce metastasis-supporting neutrophil extracellular DNA traps. Sci. Transl. Med. 361, 1-21 (2016). 
22. Denny, M. F. et al. A distinct subset of proinflammatory neutrophils isolated from patients with systemic lupus erythematosus induces vascular damage and synthesizes type I IFNs. J. Immunol. 184, 3284-3297 (2010).

23. Jenne, C. N. et al. Neutrophils recruited to sites of infection protect from virus challenge by releasing neutrophil extracellular traps. Cell Host Microbe 13, 169-180 (2013).

24. Kessenbrock, K. et al. Netting neutrophils in autoimmune small-vessel vasculitis. Nat. Med. 15, 623-625 (2009).

25. Liew, P. X. \& Kubes, P. The Neutrophil's role during health and disease. Physiol. Rev. 99, 1223-1248 (2018).

26. Okubo, K. et al. Lactoferrin suppresses neutrophil extracellular traps release in inflammation. EBioMedicine 10, 204-215 (2016).

27. Sørensen, O. E. \& Borregaard, N. Neutrophil extracellular traps - the dark side of neutrophils. J. Clin. Invest. 126, 1612-1620 (2016).

28. Papayannopoulos, V. Neutrophil extracellular traps in immunity and disease. Nat. Rev. Immunol. 18, 134-147 (2018).

29. Hu, S. C. et al. Neutrophil extracellular trap formation is increased in psoriasis and induces human beta- defensin-2 production in epidermal keratinocytes. Sci. Rep. 6, 1-14 (2016).

30. Kahlenberg, J. M., Carmona-Rivera, C., Smith, C. K. \& Kaplan, M. J. Neutrophil extracellular trap-associated protein activation of the NLRP3 inflammasome is enhanced in lupus macrophages. J. Immunol. 190, 1217-1226 (2013).

31. Scheenstra, M. R., van Harten, R. M., Veldhuizen, E. J. A., Haagsman, H. P. \& Coorens, M. Cathelicidinsmodulate TLR-activation andinflammation. Front. Immunol. 11, 1-16 (2020).

32. Chen, L. et al. Neutrophil extracellular traps promote macrophage pyroptosis in sepsis. Cell Death Dis. 9, 1-12 (2018).

33. Clancy, D. M., Henry, C. M., Sullivan, G. P. \& Martin, S. J. Neutrophil extracellular traps can serve as platforms for processing and activation of IL-1 family cytokines. FEBS J. 284, 1712-1725 (2017).

34. Henry, C. M. et al. Neutrophil-derived proteases escalate inflammation through activation of il-36 family cytokines. Cell Rep. 14, 708-722 (2016).

35. Chen, Y. et al. Esculetin ameliorates psoriasis-like skin disease in mice by including CD4+Foxp3+ regulatory T cells. Front. Immunol. 9, 1-13 (2018).

36. Witalison, E. E., Thompson, P. R. \& Hofseth, L. J. Protein Arginine Deiminases and associated citrullination: Physiological functions and diseases associated with dysregulation. Curr Drug Targets. 16, 700-710 (2015).

37. Bicker, K. L. \& Thompson, P. R. The protein arginine deiminases (PADs): Structure, function, inhibition, and disease. Biopolymers 99, 155-163 (2013).

38. Li, P. et al. PAD4 is essential for antibacterial innate immunity mediated by neutrophil extracellular traps. J. Exp. Med. 207, 1853-1862 (2010).

39. Jorch, S. K. \& Kubes, P. An emerging role for neutrophil extracellular traps in noninfectious disease. Nat. Med. 7, 279-287 (2017).

40. Di Domizio, J. \& Gilliet, M. Psoriasis caught in the NET. J. Invest. Dermatol. 139, 1426-1429 (2019).

41. Buhl, A. L. \& Wenzel, J. Interleukin-36 in infectious and inflammatory skin diseases. Front. Immunol. 10, 1-11 (2019).

42. Boutet, M. A. et al. Distinct expression of interleukin (IL)-36a, b and c, their antagonist IL-36Ra and IL-38 in psoriasis, rheumatoid arthritis and Crohn's disease. Clin. Exp. Immunol. 184, 159-173 (2016).

43. Goldstein, J. D. et al. IL-36 signaling in keratinocytes controls early IL-23 production in psoriasis-like dermatitis. Life Sci Alliance. 3, 1-18 (2020).

44. Sutton, C. E. et al. Interleukin-1 and IL-23 induce innate IL-17 production from $\gamma \delta$ T cells, amplifying Th17 responses and autoimmunity. Immunity 31, 331-341 (2009).

45. Ishida-Yamamoto, A. et al. Decreased deiminated keratin K1 in psoriatic hyperproliferative epidermis. J. Invest. Dermatol. 114, 701-705 (2000).

46. Chavanas, S. et al. Peptidylarginine deiminases and deimination in biology and pathology: Relevance to skin homeostasis. J. Dermatol. Sci. 44, 63-72 (2006).

47. Schneider, C. A., Rasband, W. S. \& Eliceiri, K. W. NIH Image to ImageJ: 25 years of image analysis. Nat. Methods 9, 671-675 (2012).

48. Zhang, X., Goncalves, R. \& Mosser, D. M. The isolation and characterization of murine macrophages. Curr. Protoc. Immunol. 14, $1-18$ (2008).

49. Safi, R. et al. Neutrophils contribute to vasculitis by increased release of neutrophil extracellular traps in Behçet's disease. J. Dermatol. Sci. 92, 143-150 (2018).

\section{Acknowledgements}

We would like to express our gratitude to Ms. Sakai for her excellent technical assistance. This research was supported by AMED (grant number 19ek0109295H0003) and JSPS KAKENHI (grant numbers 15H04886 and 18K08281 to KS). In addition, this research was supported by grants from the Lydia O'Leary Memorial Pias Dermatological Foundation and the Maruho Takagi Dermatology Foundation to KS.

\section{Author contributions}

Conceptualization: Soichiro Watanabe, Yohei Iwata, Masashi Akiyama, and Kazumitsu Sugiura. Data curation: Soichiro Watanabe, Yohei Iwata, and Kazumitsu Sugiura. Formal analysis: Soichiro Watanabe, Yohei Iwata, Hidehiko Fukushima, Kenta Saito, and Kazumitsu Sugiura. Funding acquisition: Kazumitsu Sugiura. Investigation: Soichiro Watanabe, Yohei Iwata, Hidehiko Fukushima, Kenta Saito, Yoshihito Tanaka, Yurie Hasegawa, and Kazumitsu Sugiura. Methodology: Soichiro Watanabe, Yohei Iwata, and Kazumitsu Sugiura. Project administration: Soichiro Watanabe, Yohei Iwata, and Kazumitsu Sugiura. Resources: Soichiro Watanabe, Yohei Iwata, and Kazumitsu Sugiura. Supervision: Soichiro Watanabe, Yohei Iwata, and Kazumitsu Sugiura. Validation: Soichiro Watanabe, Yohei Iwata, and Kazumitsu Sugiura. Visualization: Soichiro Watanabe, Yohei Iwata, and Kazumitsu Sugiura. Writing - original draft: Soichiro Watanabe, Yohei Iwata, Hidehiko Fukushima, Kenta Saito, Masashi Akiyama, and Kazumitsu Sugiura. Writing - review \& editing: Soichiro Watanabe, Yohei Iwata, Hidehiko Fukushima, Kenta Saito, Yoshihito Tanaka, Yurie Hasegawa, and Kazumitsu Sugiura.

\section{Competing interests}

The authors declare no competing interests.

\section{Additional information}

Supplementary information is available for this paper at https://doi.org/10.1038/s41598-020-76864-y.

Correspondence and requests for materials should be addressed to K.S.

Reprints and permissions information is available at www.nature.com/reprints. 
Publisher's note Springer Nature remains neutral with regard to jurisdictional claims in published maps and institutional affiliations.

(c) (i) Open Access This article is licensed under a Creative Commons Attribution 4.0 International License, which permits use, sharing, adaptation, distribution and reproduction in any medium or format, as long as you give appropriate credit to the original author(s) and the source, provide a link to the Creative Commons licence, and indicate if changes were made. The images or other third party material in this article are included in the article's Creative Commons licence, unless indicated otherwise in a credit line to the material. If material is not included in the article's Creative Commons licence and your intended use is not permitted by statutory regulation or exceeds the permitted use, you will need to obtain permission directly from the copyright holder. To view a copy of this licence, visit http://creativecommons.org/licenses/by/4.0/.

(c) The Author(s) 2020 